\title{
Residual stress, intermolecular force, and frictional properties distribution maps of diamond films for micro- and nano-electromechanical (M/NEMS) applications
}

\author{
S. Gupta ${ }^{a)}$ \\ Department of Physics and Materials Science, Missouri State University, Springfield, Missouri 65897 \\ O.A. Williams \\ Institute for Materials Research, Universiteit Hasselt, BE-3590 Diepenbeek, Belgium \\ R.J. Patel \\ Department of Physics and Materials Science, Missouri State University, Springfield, Missouri 65897 \\ K. Haenen \\ Institute for Materials Research, Universiteit Hasselt, BE-3590 Diepenbeek, Belgium
}

(Received 11 May 2006; accepted 3 August 2006)

\begin{abstract}
Carbon in its various forms, specifically nanocrystalline diamond, may become a key material for the manufacturing of micro- and nano-electromechanical (M/NEMS) devices in the twenty-first century. To utilize effectively these materials for M/NEMS applications, understanding of their microscopic structure and physical properties (mechanical properties, in particular) become indispensable. The microcrystalline and nanocrystalline diamond films were grown using hot-filament and microwave chemical vapor deposition techniques involving novel $\mathrm{CH}_{4} /\left[\mathrm{TMB}\right.$ for boron doping and $\mathrm{H}_{2} \mathrm{~S}$ for sulfur incorporation] in high hydrogen dilution chemistry. To investigate residual stress distribution and intermolecular forces at nanoscale, the films were characterized using Raman spectroscopy and atomic force microscopy in terms of topography, force curves, and force volume imaging. Traditional force curve measures the force felt by the tip as it approaches and retracts from a point on the sample surface, whereas force volume is an array of force curves over an extended range of sample area. Moreover, detailed microscale structural studies are able to demonstrate that the carbon bonding configuration ( $\mathrm{sp}^{2}$ versus $\mathrm{sp}^{3}$ hybridization) and surface chemical termination in both the un-doped and doped diamond have a strong effect on nanoscale intermolecular forces. The preliminary information in the force volume measurement was decoupled from topographic data to offer new insights into the materials' surface and mechanical properties of diamond films. These measurements are also complemented with scanning electron microscopy and x-ray diffraction to reveal their morphology and structure and frictional properties, albeit qualitative using lateral force microscopy mode. We present these comparative results and discuss their potential impact for electronic and electromechanical applications.
\end{abstract}

\section{INTRODUCTION}

Diamond is a promising wide-gap semiconductor material with a large potential offering excitement and interest due to its unique blend of superlative physical (electronic, optical, mechanical, and chemical) properties. ${ }^{1,2}$ Diamond thin film (DTF) are attractive for several applications such as in tribological coatings and cutting

\footnotetext{
a) Address all correspondence to this author.

Present address: Univ. of Missouri, ECE Department, Columbia, MO 65211-2300.

e-mail: guptas@missouri.edu

DOI: $10.1557 / J M R .2006 .0372$
}

tools (extreme hardness), heat sinks ${ }^{3,4}$ (high thermal conductivity), optical windows (wide band gap, $5.45 \mathrm{eV})^{5}$ (wide spectral transparency), high temperature and high power electronics (breakdown voltage of $\sim 10^{7} \mathrm{~V} / \mathrm{cm}$ ), biosensors ${ }^{6}$ (chemical inertness), and vacuum microelectronics such as field-emission arrays, ${ }^{7}$ and therefore it is considered an engineering material. These previously mentioned properties are undoubtedly unmatchable by any other material and it is thus apparent that diamond is a material of choice. Intense research efforts over the past two decades have yielded the technology to grow high-quality diamond thin films on non-diamond substrates ${ }^{8,9}$ thus enabling some of these applications mentioned previously. 
A variety of diamond chemical vapor deposition (CVD) techniques currently available use different activation sources to create plasma (a soup of radicals and neutrals) include: thermal (hot filament, flame), radiation [(rf, microwave (MW)], and electric fields (dc plasma) and others. ${ }^{10}$ Although, these deposition techniques share some characteristics, each one of them has its own set of optimized processing conditions that can be used to produce films with different sets of structural and physical properties. However, these films are rougher $(\sim 100$ $200 \mathrm{~nm}$ rms) with micron-size crystallites and contain non-negligible amount of $\mathrm{sp}^{2}$-bonded carbon $\left(\mathrm{sp}^{2} \mathrm{C}\right)$. A considerable effort is currently devoted to understand and control the surface morphology (textured versus fine-grained) and film composition $\left(\mathrm{sp}^{3} \mathrm{C}\right.$ versus $\mathrm{sp}^{3} \mathrm{C} /$ $\mathrm{sp}^{2} \mathrm{C}$ ) of these films because these features substantially affect their physical (mechanical and electromechanical, in particular) properties. Attempts to obtain relatively smooth films usually result in a considerable increase in $\mathrm{sp}^{2}$-bonded carbon $\left(\mathrm{sp}^{2} \mathrm{C}\right)$. Therefore, smooth diamond films with negligible amount of $\mathrm{sp}^{2} \mathrm{C}$ are highly desirable. ${ }^{11}$ Nanocrystalline diamond, where the grain size is a few nanometers, is quite promising in connection to the goal of achieving highly transparent smoother surfaces in addition to preserving a high degree of many fundamental properties (mechanical hardness, chemical inertness, and biological compatibility). ${ }^{12,13}$ Thus they are attractive for $\mathrm{x}$-ray lithography masks, ${ }^{14}$ extreme hardness used for coating tools and sliding parts, ${ }^{1}$ high field emission for flat panel displays (FPDs), ${ }^{15} \mathrm{SAW}$ devices, micro-fluidics, and nano-/micro-electromechanical systems (N/MEMS). ${ }^{16,17}$ Usually several properties of these materials are attributed to the relative ratio and the spatial correlation or connectivity of $\mathrm{sp}^{3} / \mathrm{sp}^{2}$ coordinated carbon. ${ }^{18}$ Therefore significance is given to the identification of the microscale structure to establish "propertystructure" correlation from a technological standpoint.

Doping in diamond to make it semiconducting (both pand n-type) is currently another subject of investigation. It is because electrically diamond is a wide band gap semiconductor and limited by the fact that only p-type diamond is readily available through Boron doping. Apart from the fundamental interests, n-type diamond finds many applications and availing n-type diamond is still a major open topic of research. ${ }^{19}$ In analogy to the conventional dopants used in $\mathrm{Si}$, elements of group $\mathrm{V}$ (phosphorus and nitrogen) and VI (sulfur) have been studied in diamond. ${ }^{20}$ Hereby, the idea to introduce sulfur both in microcrystalline and nanocrystalline diamond was not only fundamental but rather to introduce nanoscale heterogeneity for electronic display applications. ${ }^{21}$ We have deposited both microcrystalline and nanocrystalline diamond films by hot-filament chemical vapor deposition (HFCVD) and microwave chemical vapor deposition (MWCVD) techniques and investigated the structural and mechanical properties with p- and n-type doping. This research illustrates a broad range of carbonbased materials and provides some qualitative understanding of mechanical properties through surface probe microscopy as to how to engineer them via synthesis parameters. Moreover, this paper reports some of the interesting findings, which would relate and highlight some of the differences or similarities in our material and the existing ones in the literature.

\section{EXPERIMENTAL DETAILS}

Diamond thin films used in the present study were synthesized using hot-filament (HFCVD) and microwave chemical vapor deposition (MWCVD) techniques on 1 -in. Mo disk and $\mathrm{Si}(001)$ as substrates using methane in high hydrogen dilution in addition to boron and sulfur for comparative studies. While boron doped samples were deposited by MWCVD, the HFCVD technique was used to grow sulfur doped diamond films. The substrates were scrubbed with $0.1 \mu \mathrm{m}$ synthetic diamond powder (Alfa Aesar, Ward Hill, MA) to provide nucleation and then all of the substrates were ultrasonically cleaned thoroughly in methanol for $15 \mathrm{~min}$. They were then dried in $\mathrm{N}_{2}$ and placed immediately on a molybdenum substrate holder that is integrated with a graphite heater and induction heater in HFCVD and MWCVD reactors, respectively. ${ }^{22,23}$

Briefly, before introducing the clean reactive gas mixture, the chamber was evacuated to $10^{-7}$ Torr or lower. The gas flow of methane and hydrogen were controlled to obtain methane concentrations of $0.3-2.0 \% .{ }^{24}$ In the HFCVD, the gas mixture was activated through a heated Rhenium (Re) filament positioned at $8 \mathrm{~mm}$ above the heated Mo disk used as substrate. The total gas pressure of the chamber was kept around 20-50 Torr. The substrate temperature was kept at $750{ }^{\circ} \mathrm{C}$. Besides the previously mentioned conditions, to introduce sulfur (S) in the samples, hydrogen sulfide $\left(\mathrm{H}_{2} \mathrm{~S}, 500 \mathrm{ppm}\right)$ :hydrogen $\left(\mathrm{H}_{2}\right)$ gas mixture was introduced in the chamber along with $\mathrm{CH}_{4}: \mathrm{H}_{2}$ mixture as feedstock gas. For boron doping, trimethylborate (TMB) of $1000 \mathrm{ppm}$ in gas phase was used in MWCVD reactor. ${ }^{24}$ The details of the growth parameters are summarized in Table I.

Film thicknesses were around $1.5 \mu \mathrm{m}$ for all of the samples. The surface morphology was revealed from SEM (JEOL 3400, Sheboygan, WI), and the average grain size and surface roughness were estimated using AFM (Digital Instrument, Model Dimension 3100). The XRD measurements were taken by Siemens D2000 diffractometer using a Cu- $\mathrm{K}_{\alpha}$ line source $\left(\lambda_{\mathrm{L}}=1.5405 \AA\right.$ ), in a $\theta-2 \theta$ configuration to probe the structure. Raman spectroscopy (RS) is used to analyze the structural phases and the bonding configurations in the films. The microRaman spectra were recorded with a J-Y TRIAX 320 spectrometer working in the backscattered configuration. 
TABLE I. Deposition parameters for poly/microcrystalline and nanocrystalline diamond films by chemical vapor deposition techniques.

\begin{tabular}{|c|c|c|}
\hline \multicolumn{2}{|l|}{ Parameters } & \multirow[b]{2}{*}{ MWCVD } \\
\hline & $\mathrm{HFCVD}^{\mathrm{a}}$ & \\
\hline Substrate & Si (001) and Mo & n-Si (001) \\
\hline Filament temperature $\left(\mathrm{T}_{\mathrm{f}}\right)$ & $2500^{\circ} \mathrm{C}$ & $\cdots$ \\
\hline Filament length & $8 \mathrm{~cm}$ & $\cdots$ \\
\hline Filament-substrate distance & $8 \mathrm{~mm}$ & $\cdots$ \\
\hline Total pressure of the chamber $(\mathrm{P})$ & 20.0 Torr & 50.0 Torr \\
\hline Substrate temperature $\left(\mathrm{T}_{\mathrm{S}}\right)$ & $700{ }^{\circ} \mathrm{C} / 900{ }^{\circ} \mathrm{C}$ & $850^{\circ} \mathrm{C}$ \\
\hline Source gases & $\mathrm{CH}_{4} / \mathrm{H}_{2} / \mathrm{H}_{2} \mathrm{~S}$ & $\mathrm{CH}_{4} / \mathrm{H}_{2} / \mathrm{TMB}$ \\
\hline Total gas flow rate & $100 \mathrm{sccm}$ & $100 \mathrm{sccm}$ \\
\hline $\mathrm{H}_{2}$ & $80 \mathrm{sccm}$ & $80.0 \mathrm{sccm}$ \\
\hline $\mathrm{CH}_{4} / \mathrm{H}_{2}$ & $20 \mathrm{sccm}$ & $20.0 \mathrm{sccm}$ \\
\hline Methane concentration & $0.3-2.0 \%$ & $2.0 \%$ \\
\hline $\mathrm{H}_{2} \mathrm{~S}$ & 500 ppm & $\cdots$ \\
\hline TMB in gas phase & $\cdots$ & 1000 ppm \\
\hline Deposition time & $15-120$ minutes & 120 minutes \\
\hline Microwave power & $\cdots$ & $1300 \mathrm{~W}$ \\
\hline
\end{tabular}

${ }^{\mathrm{a}}$ HFCVD $=$ hot-filament chemical vapor deposition; MWCVD $=$ microwave chemical vapor deposition.

The $514.5 \mathrm{~nm}$ line of an $\mathrm{Ar}^{+}$laser was used as excitation source with 5-10 $\mathrm{mW}$ incident laser power to avoid thermal effects. Since the spectra were recorded using a $50 \times$ objective, the probed area was about $3-4 \mu \mathrm{m}^{2}$. To investigate residual stress distribution and to measure elasticity maps on these surfaces, the films were characterized extensively using Raman spectroscopy in an area of $20 \times$ $20 \mu \mathrm{m}^{2}$ and atomic force microscopy in terms of force curves with the latter measuring elasticity maps, alternatively intermolecular forces between the tip and a surface. Traditional force curve measures the force felt by the tip as it approaches and retracts from a point on the sample surface, while force volume is an array of force curves over an extended range of sample area. ${ }^{25}$ Moreover, by using atomic force microscopy for nanoscale force constant measurements and surface spectroscopy techniques for detailed chemical and structural studies, we are able to demonstrate that the carbon bonding configuration $\left(\mathrm{sp}^{2}\right.$ versus $\mathrm{sp}^{3}$ hybridization) and surface chemical termination in both the un-doped and doped diamond surfaces has a strong effect on nanoscale intermolecular forces. The preliminary information obtained from the force volume measurements was decoupled from topographic data to offer new insight into the materials' surface properties. Two samples of each kind were measured several times and the results were reproducible within an accuracy of $2-4 \%$.

\section{RESULTS AND DISCUSSION}

\section{A. Morphological and structural properties}

Figure 1 shows SEM micrographs of microcrystalline diamond films both the un-doped [Fig. 1(a)] and doped with boron [Fig. 1(b)] and sulfur [Fig. 1(c)] revealing surface morphology. Notice that, while the un-doped and boron-doped diamond films were synthesized using MWCVD, the HFCVD was used to deposit sulfurincorporated diamond films. The SEM micrographs show that the films are composed of uniformly densely packed well-faceted diamond crystallites (with triangular growth habit or $\langle 111\rangle$ facets) for un-doped that start to turn into a finely grained non-faceted texture on doping. However, the effect is more pronounced for sulfur doping than that of boron doping. The AFM analysis indicated that the average grain size varied from $0.5 \mu \mathrm{m}$ for the un-doped and 0.4 and $0.2 \mu \mathrm{m}$ for the sulfur and boron doped diamond films. The variation of grain sizes and the rms surface roughness $(\sigma)$ values evaluated using AFM (Fig. 1) is roughly varied between $400-100 \mathrm{~nm}$ and 120 $60 \mathrm{~nm}$, respectively. Notice that the boron and sulfur addition reduces the surface roughness. In comparison to $\mathrm{N}$ addition, the thermodynamic calculations suggested that they induce a transition from faceted to un-faceted morphology by activated species (possibly HCB and $\mathrm{HCS}$ analogous to $\mathrm{HCN}$ ). Figure 2 shows XRD diffractogram for the representative samples as mentioned previously depicting the characteristic XRD diamond (111) peak at $2 \theta=44^{\circ}$. Qualitatively, the full width at halfmaximum (FWHM) of the characteristic XRD diamond peak becomes broader and relatively less intense with boron and sulfur incorporation (Fig. 2). Also, it shifts toward higher $2 \theta$ values, indicating the development of compressive stress in the films. The corresponding crystallite sizes were estimated using Debye-Scherrer method: $\left[L_{h k l}=(K \lambda / b \cos \theta)\right]$ where $\mathrm{K} \sim 1$ (DebyeScherrer constant), $\lambda=1.5405 \AA\left(\mathrm{Cu} \mathrm{K} \mathrm{K}_{\alpha}\right)$, and $\mathrm{b}$ is the FWHM of $\langle 111\rangle$ peak. ${ }^{26}$ The FWHM values for the XRD peak ranged between $0.033^{\circ}-0.07^{\circ}$ and the calculated crystallite size is in good agreement with the ones estimated using AFM grain size analysis. The peak appearing at around $26^{\circ}$ and $/$ or $40^{\circ}$ in the XRD scans indicating the existence of a $\mathrm{SiC}$ interlayer was present in the samples.

\section{B. Microstructural properties}

Raman spectroscopy (RS) has emerged as one of the most frequently used tools to characterize carbon-based materials, in general. ${ }^{27,28}$ Since Raman can detect changes in behavior of $\mathrm{C}-\mathrm{C}$ bonds, probing microstructural variation due to any treatment is much more informative and quantitative. Visible $\mathrm{RS}\left(\lambda_{\mathrm{L}}=514.5 \mathrm{~nm}\right.$ or $\mathrm{E}_{\mathrm{L}}=2.41 \mathrm{eV}$ ) used hereby is used to assess the crystalline quality and to probe the changes in the structural bonding configurations with respect to doping. In addition, Raman scattering depends on the electronphonon interactions, which are sensitive to local environments. To enable the technological applications mentioned previously, we all know that specific sets of 

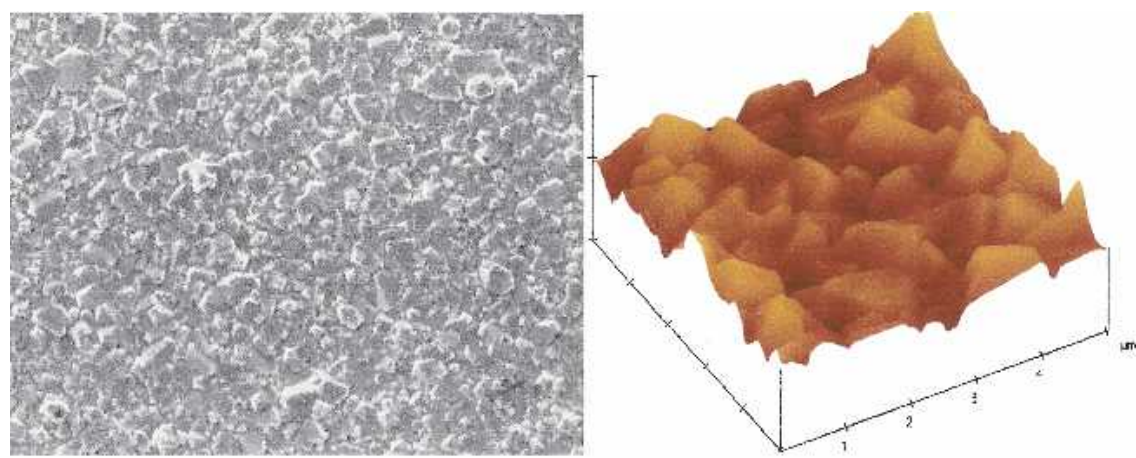

(a)
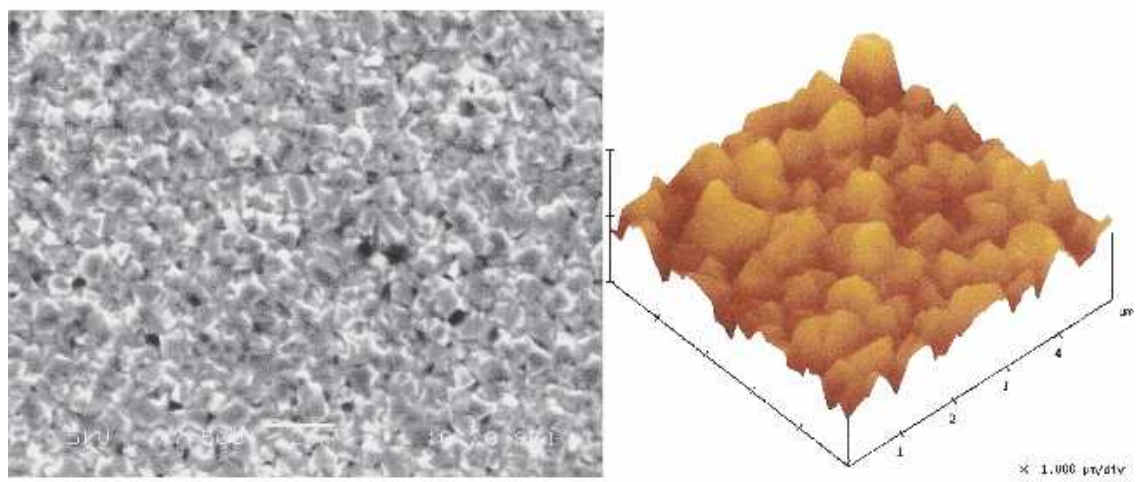

(b)
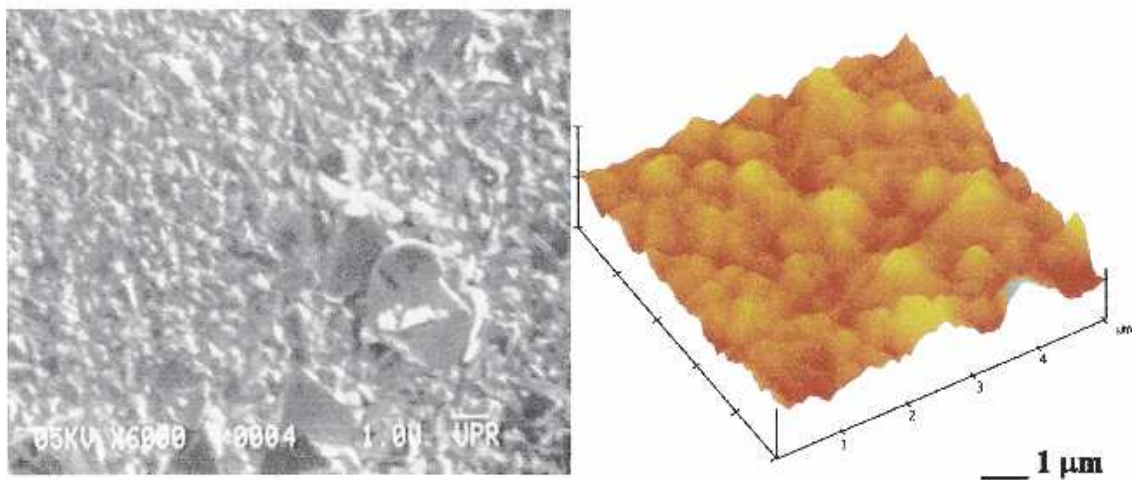

(c)

FIG. 1. Scanning electron micrographs of (a) un-doped, (b) boron-doped, and (c) sulfur-incorporated poly/microcrystalline diamond films depicting the change in surface morphology and corresponding three-dimensional atomic force microscopy images. Whereas the un-doped and boron-doped diamond films were synthesized using MWCVD, the HFCVD was used to deposit sulfur-incorporated diamond films.

material characteristics must be achieved, thus requiring control of both the microstructure and microstrain in the diamond films. To evaluate these properties, we used micro-Raman spectroscopy and we not only determined individual crystalline quality, diamond content, or $\mathrm{sp}^{3}$-bonded carbon fraction and the total stress, but also mapped over an area of $20 \times 20 \mu \mathrm{m}^{2}$ thus providing areal distribution of all of these quantities. The diamond content was evaluated using the following equation: $f_{s p 3 C}(\%)=\left(50 * I_{d} / 50 * I_{d}+I_{n d}\right) \times 100$, which is the ratio of the diamond peak area (integrated intensity) to that of the rest of the spectrum between $1100-1700 \mathrm{~cm}^{-1}$ (taking the Raman scattering cross-section of non-sp ${ }^{3}$ bonded carbon to be 50 times that of diamond). ${ }^{29}$ Similarly the microstress/strain is calculated from the Raman shift using the pressure coefficient of the diamond Raman peak: $-1.9 * \sigma_{\text {comp }}(G P a)=\left(\omega-\omega_{0}\right)$; where $\omega_{0}=$ $1332 \mathrm{~cm}^{-1}$ for a single crystal diamond. ${ }^{30}$ Table II summarizes the lower and upper bounds of those values.

Figure 3 shows Raman spectra of the diamond films shown in Fig. 1. Qualitative inspection of Fig. 3 reveals that the incorporation of boron and sulfur to the CVD process enhances the formation of non-diamond carbon (i.e., primarily $\mathrm{sp}^{2}$-bonded carbon; $\mathrm{sp}^{2} \mathrm{C}$ ) within the poly/microcrystalline diamond films, in addition to the characteristic diamond Raman signature at $1332.5 \mathrm{~cm}^{-1}$, 

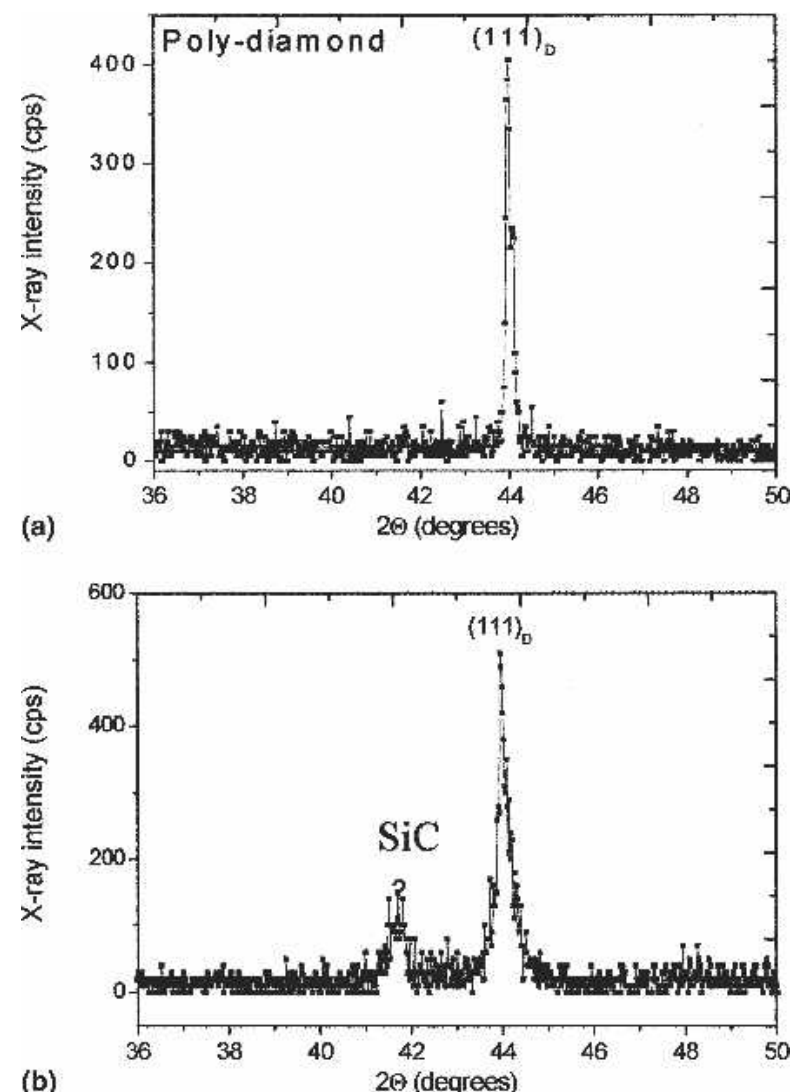

(b)

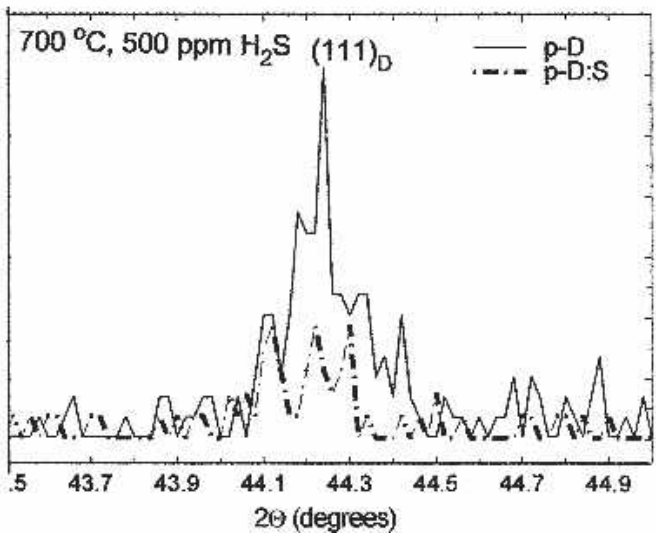

(c)

FIG. 2. Typical x-ray diffractograms of (a) un-doped, (b) borondoped, and (c) sulfur-incorporated poly/microcrystalline diamond films depicting characteristic diamond peak (111) occurring at $2 \theta$ $\sim 44.00^{\circ}$.

TABLE II. Summary of spatial distribution of diamond fraction and residual stress mapped using Raman spectroscopy.

\begin{tabular}{lcccc}
\hline \hline $\begin{array}{c}\text { Sample } \\
\text { ID }\end{array}$ & $\begin{array}{c}\mathrm{sp}^{3} \mathrm{C} \\
\text { concentration } \\
(\%)\end{array}$ & $\begin{array}{c}\text { Stress } \\
(\mathrm{Gpa})-\sigma_{\text {comp }}\end{array}$ & $\begin{array}{c}\text { q or [B] } \\
\left(\mathrm{cm}^{-3}\right)\end{array}$ & $\begin{array}{c}\text { Intrinsic stress } \\
(\mathrm{Gpa})-\sigma_{\text {int }}\end{array}$ \\
\hline Un-doped & $90-98$ & $1.1-2.1$ & $\ldots$ & $0.5-1.5$ \\
B-doped & $94-97$ & $0.7-1.52$ & $>10^{19}$ & $0.1-0.9$ \\
S-doped & $47-96$ & $1.66-3.05$ & $\cdots$ & $1.1-2.9$ \\
\hline \hline
\end{tabular}
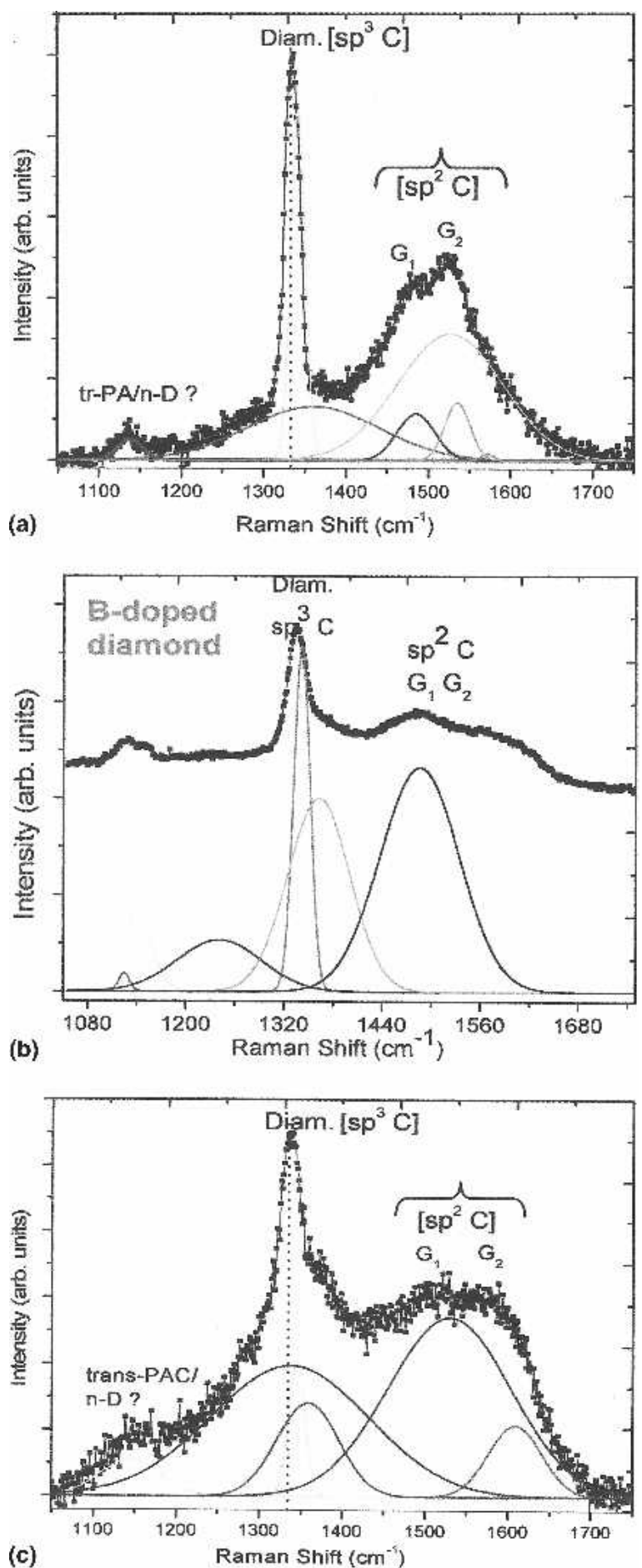

FIG. 3. Typical Raman spectra of (a) un-doped, (b) boron-doped, and (c) sulfur-incorporated poly/microcrystalline diamond films depicting the characteristic diamond $\left(\mathrm{sp}^{3} \mathrm{C}\right)$ and graphitic $\left(\mathrm{sp}^{2} \mathrm{C}\right)$ signatures.

which is the fingerprint of diamond $\left(\mathrm{sp}^{3}\right.$-bonded carbon; $\mathrm{sp}^{3} \mathrm{C}$ ) and it appears to be broadened. ${ }^{31}$ The $\mathrm{sp}^{3} \mathrm{C}$ bonds in the films amount to $>90 \%$ for the un-doped and Bdoped films, while reduced to $>75 \%$ for the sulfur-doped. It is thus apparent that sulfur tends to introduce more 
disorder in the films. The full width at half- maximum (FWHM) denoted by $\Gamma$ of the characteristic diamond peak is a qualitative measure of the perfection of the diamond crystal structure and the intensity ratio of the diamond peak to the rest of the spectrum provides a measure of $\mathrm{sp}^{3} \mathrm{C}$ fraction. It is generally believed that a broadened diamond peak characterizes the diamond with defects/disorder. On quantitative evaluation, it was found that though the FWHM values do vary with the deposition parameters ranging 2.9-5.3, it is rather difficult to evaluate the quality of the films, in general. ${ }^{32}$

Figures 4(a)-4(c) show the spatial mapping generated successfully using Raman spectroscopy for the un-doped, B-doped, and S-incorporated diamond films, respectively providing an insight about uniformity/homogeneity, stress distribution, and corresponding microstructure.
These findings are tabulated in Table II. The excess sulfur atoms in the latter act as impurities within microcrystalline diamond and they have a tendency to aggregate within grain boundaries unlike boron, which acts as a traditional $p$-type dopant substituting carbon and thus preserving the structural integrity of the material to a greater extent. ${ }^{23,33}$

\section{Intermolecular force and frictional property characterizations}

In addition to surface topography, the atomic force microscopy is used to probe intermolecular forces between the tip and the surface. It is usually performed by recording the cantilever deflection as the tip approached a surface and then plotting a force curve as a function of

(a)
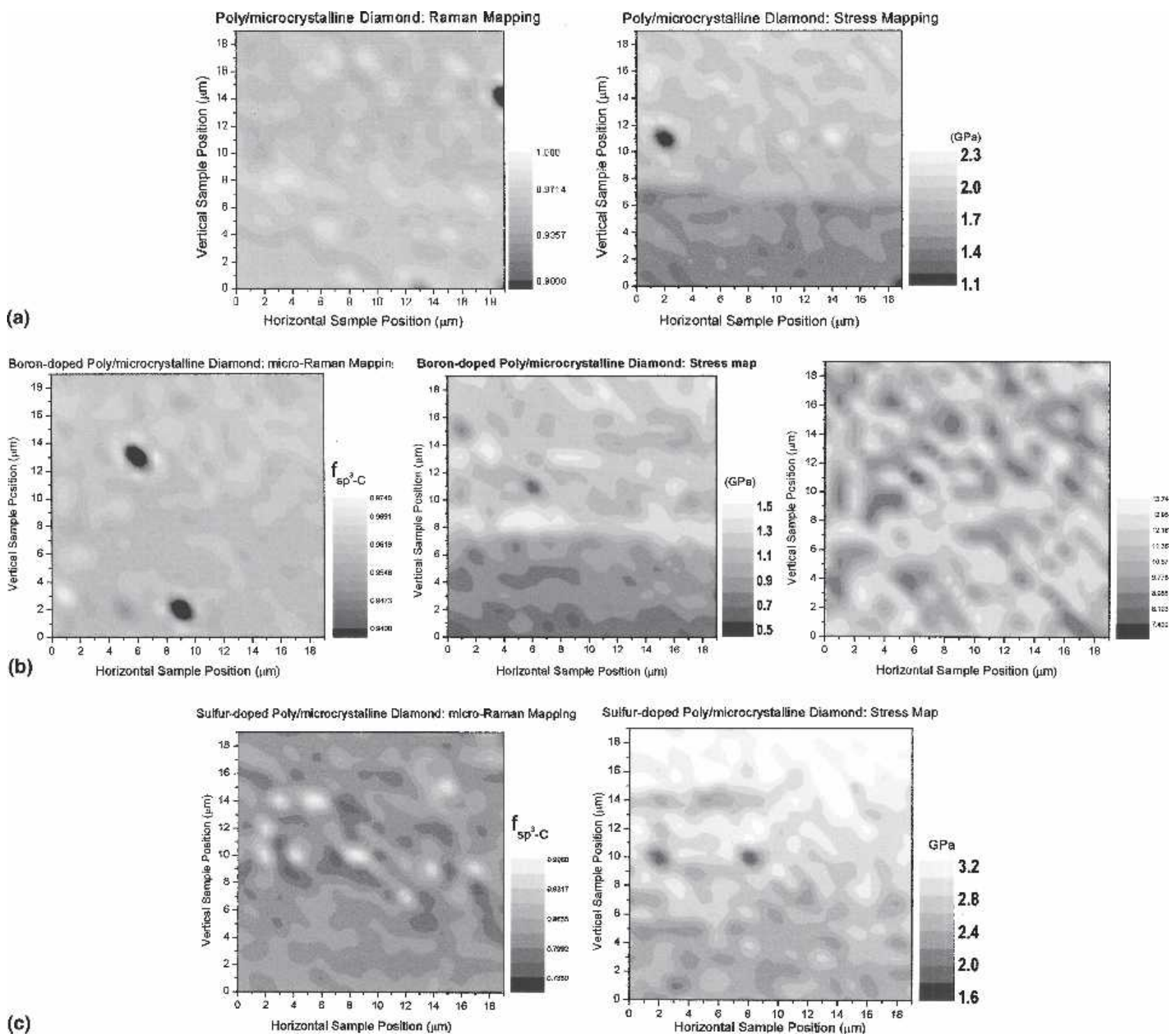

(c)

FIG. 4. Shown are the $\mathrm{sp}^{3}$-bonded carbon and residual stress distribution maps in the area of $20 \times 20 \mu \mathrm{m}^{2}$ for (a) un-doped, (b) boron-doped, and (c) sulfur-incorporated poly/microcrystalline diamond films deduced from Raman spectra. 
distance between the tip and surface thus providing information about the electrostatic, chemical, and magnetic properties of surfaces. ${ }^{34-37}$ Since most of the surfaces are not homogenous, it is often of interest to collect an array of force curves; so-called force sampling or force volume/imaging. Such an array produces information about the lateral or spatial distribution of different surfaces and materials properties. Figures 5(a)-5(c) show the force volume imaging for the diamond films as shown in Fig. 1. Apparent from the force volume imaging and corresponding force curves at different points on the surface of the films shown in Figs. 5(a)-5(c) is that the un-doped diamond has far less in-homogeneity than those of boron and is further increased in the sulfurincorporated films. Since force is the derivative of energy with respect to distance, the volume data can be used to infer the potential energy between surfaces. Recalling that the contact force $F=k(\Delta Z)$ where $k$ is the spring contact of the tip, one can estimate the contact force from the force plots. In all of the microcrystalline diamond films, the interaction force seems to be largely dominated by strong adhesion, which is usually characterized by the tip retract curve shape. ${ }^{35,38}$ However, we also showed forced volume curves/imaging for un-doped and sulfurincorporated [Figs. 5(d) and 5(e)] nanocrystalline diamond for comparative studies.

The nanocrystalline diamond films are highly dense continuous films with no distinctive growth morphology [Figs. 5(d) and 5(e)] in contrast to the columnar morphology for microcrystalline diamond. This occurs due to the increase in the heterogeneous nucleation rate and consequent decrease in the crystallite size. ${ }^{11}$ Because of the resulting average grain size, which is below hundreds of nanometers, they are described as nanocrystalline diamond. Consistent with the results, the rms surface roughness $(\sigma)$ estimated from AFM decreases with increasing sulfur addition from around $120 \mathrm{~nm}$ to $15 \mathrm{~nm}$ [Figs. 5(d) and 5(e)]. The crystallite size distribution using AFM grain size analysis followed a normal distribution ranging 15-20 $\mathrm{nm}$. Hence, the S-incorporated films show
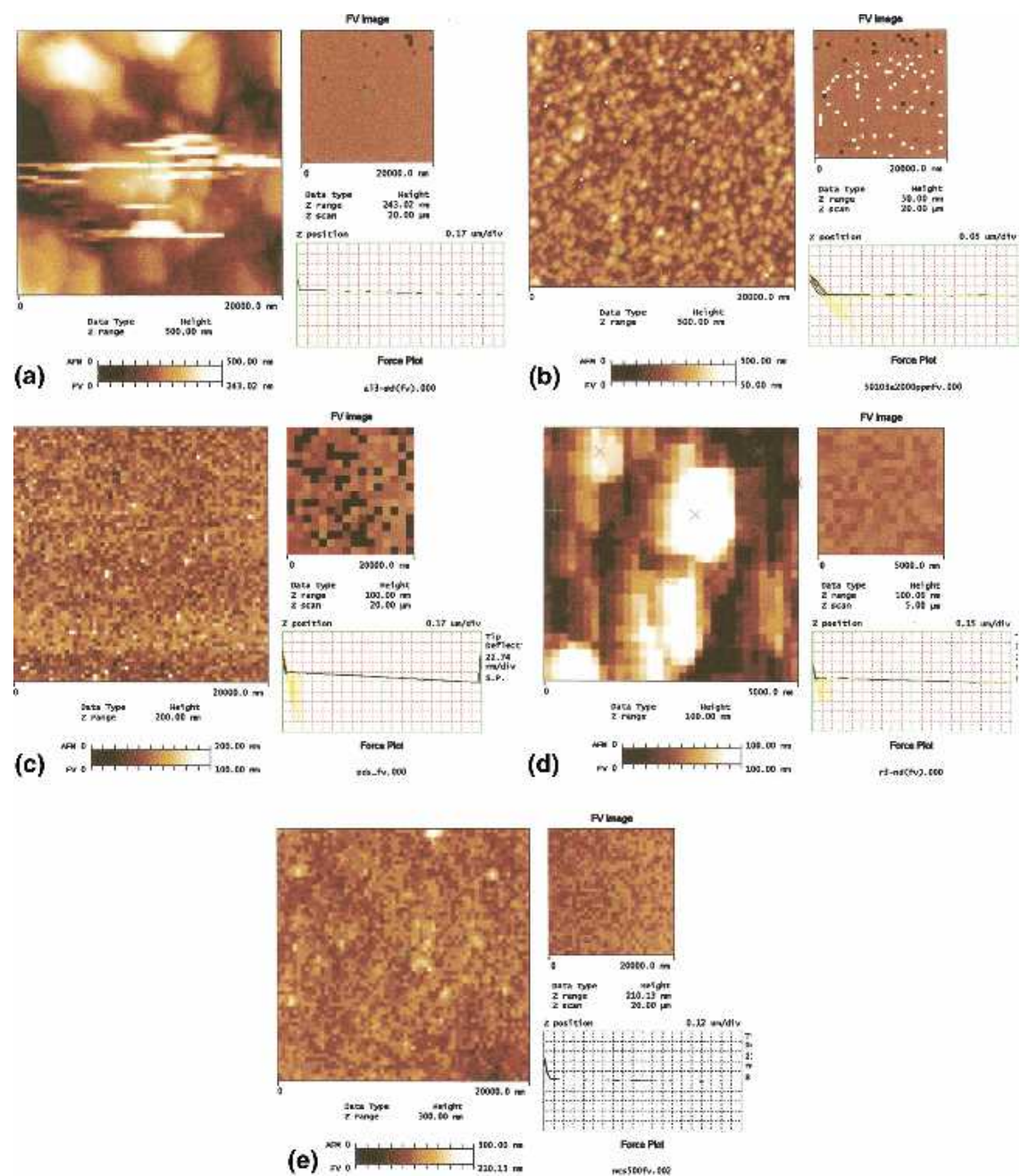

FIG. 5. Shown are the force volume curves measuring elasticity maps for (a-e) un-doped, boron-doped, and sulfur-incorporated poly/ microcrystalline and nanocrystalline diamond films, respectively measured using atomic force microscopy. 
smoother surfaces and smaller grains than those grown without sulfur. The Raman spectra for the sulfurincorporated nanocrystalline diamond (not shown, please refer to Ref. 22) are very different to those obtained for high-quality poly/microcrystalline diamond films, due to the selection rule breakdown, thus revealing drastic microstructural changes in the films. Apart from the wellknown characteristic diamond peak at $1332.5 \mathrm{~cm}^{-1}$ indicating the existence of diamond crystallites, the Raman spectra are dominated by other contributions: (i) broad structure located around $1510 \mathrm{~cm}^{-1}$ attributed to graphitic carbon ( $\mathrm{G}$ band) that shifts to $1580 \mathrm{~cm}^{-1}$ as the graphitic clusters become more organized ${ }^{39}$; (ii) a broader structure at $1360 \mathrm{~cm}^{-1}$ (D band) attributed to aromatic clusters; and (iii) a broad shoulder present at around $1150 \mathrm{~cm}^{-1}$, which is an indication of $\mathrm{sp}^{2} /$ hydrogen/trans-polyacetylene $e^{40,41}$ and is no longer a subject of debate. The non-diamond carbon in such materials refers to a variety of $\mathrm{sp}^{3}$ - and $\mathrm{sp}^{2}$-bonded carbon materials, which can coexist in a dispersed or clustered state and in a large range of relative concentrations, depending on deposition or processing conditions or even both.

The behavior of the Raman features of the films are indicating that by stepwise departing from the optimum methane concentration $(0.3 \%)$ for the deposition of poly/ microcrystalline diamond to up to $2 \%$ along with sulfur addition, the grown films become nanocrystalline diamond-composites of $\mathrm{sp}^{2}$-bonded carbon materials (graphitic and polymeric, in either a dispersed or clustered state) within an $\mathrm{sp}^{3}$-bonded carbon matrix. It also shows that sulfur tends to introduce disorder and defects considerably, similar to nitrogen or phosphorus incorporation, which induces graphitization of carbon films. Broadening of the diamond peak to the extent that it is almost convoluted with the D band because of the reduction in the crystallite size to $\mathrm{nm}$ scale, with the concomitant development of the scattering intensity in the $1400-1600 \mathrm{~cm}^{-1}$ region (G-band), further pointing at the increase of $\pi$-bonded nature of the films (i.e., $\mathrm{sp}^{2} \mathrm{C}$ ). ${ }^{21}$

Similar to microcrystalline diamond, the force curves and corresponding force volume imaging of nanocrystalline diamond are qualitatively interpreted, which are useful in determining the adhesion and hardness characteristics of the samples and allows imaging of interaction forces. Both the un-doped and S-incorporated nanocrystalline diamond sample seems to preserve large adhesion and hence are comparable. Nonetheless, we realize that more investigations are needed.

Lateral force microscopy (LFM) is an extension of contact mode in atomic force microscopy, whereby an additional detected parameter is the torsion of the cantilever that changes depending on the friction force. The LFM can provide material-sensitive contrast since different components of a composite material exert different friction forces on the scanning tip. The technique of LFM also helps for obtaining edge-enhanced images. Figure 6 shows the frictional coefficient maps for the un-doped [Fig. 6(a)], boron-doped [Fig. 6(b)] and sulfur-incorporated [Fig. 6(c)] microcrystalline diamond. Figures 6(d) and 6(e) are for the as-deposited and sulfurincorporated nanocrystalline diamond films. There seems

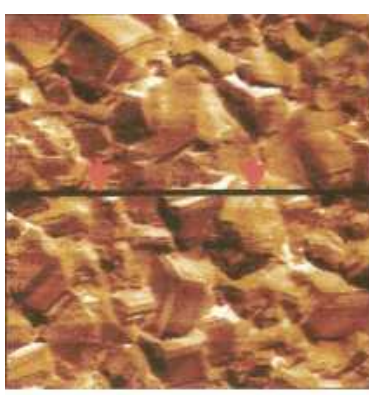

(a)

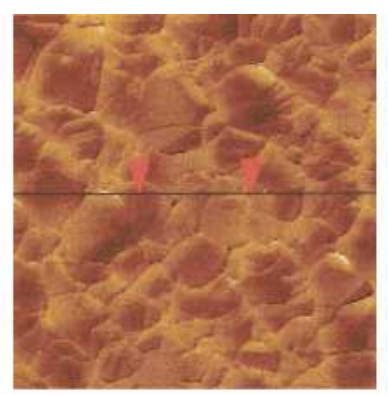

(b)

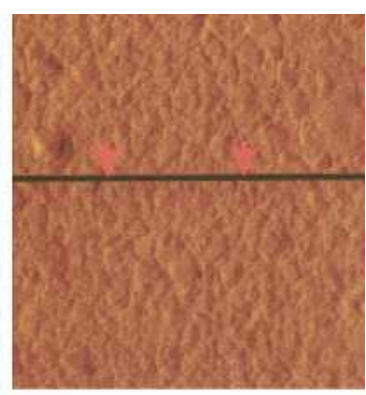

(c)

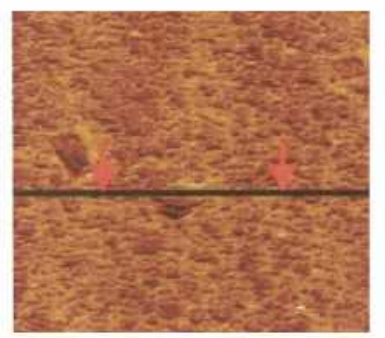

(d)

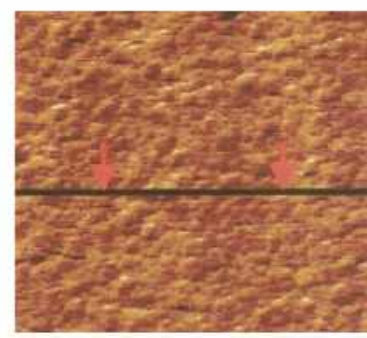

(e)

FIG. 6. Shown are the lateral force microscopy images demonstrating the friction coefficient distribution for (a-e) un-doped, boron-doped, and sulfur-incorporated poly/microcrystalline and nanocrystalline diamond films, respectively. 
to be an apparent compositional difference among all of these samples, which tallies fairly well with those assessed using micro-Raman spectroscopy. Nevertheless, it is difficult to say clearly about the coefficient of friction and for the microcrystalline diamond, in particular. It is because the frictional characteristics are obscured by the rough topography unlike those of doped and nanocrystalline diamond surfaces that are relatively smoother. By definition, $\mu=\left(f_{\mathrm{L}} / f_{\mathrm{N}}\right)$, where $f_{\mathrm{L}}$ is the lateral force and $f_{\mathrm{N}}$ the normal force. Therefore, to assess rather qualitatively we adopted the section analysis approach as shown in Fig. 6 with a horizontal line with arrows and determined $\mu$ distribution, which comes out to be in the following order: $\mu_{\text {poly/micro }}>\mu_{B}>\mu_{S}>\mu_{\text {nano }}$ $>\mu_{\text {nanos }}: 0.7-0.02$ and corresponding surface roughness is $\sigma_{\text {poly } / \text { micro }}>\sigma_{\mathrm{B}}>\sigma_{\mathrm{S}}>\sigma_{\text {nano }}>\sigma_{\text {nanos }}: 120-10 \mathrm{~nm}$. The coefficient of friction for nanocrystalline diamond films reduced by more than one order of magnitude and therefore may prove to be a potential candidate for the proposed M/NEMS devices.

\section{CONCLUSIONS}

The novel nanocrystalline diamond films may provide a basis for the revolutionary N/MEMS. Nevertheless, to effectively utilize these materials for these applications, understanding of their structural and physical properties (mechanical properties, in particular) becomes indispensable. We reported detailed and systematic study of the spatial distribution of carbon bonding and residual stress using Raman spectroscopy and intermolecular forces and friction coefficient on these surfaces using atomic force microscopy in force volume imaging and lateral force microscopy modes. The effects of boron and sulfur doping over the film microstructure for both the microcrystalline and nanocrystalline diamond is presented and the previously mentioned properties are qualitatively elucidated. These results were also complemented with the SEM and XRD techniques. The surface structure in both the un-doped and doped diamond films has a strong effect on intermolecular forces and frictional properties. The preliminary information in the force volume measurement was decoupled from topographic data to offer new insight into the materials and surface properties. These findings will be further investigated albeit quantitatively using tribological properties, for instance and will be published in subsequent reports for nano-/microelectromechanical systems. Through these studies we also obtained an integral picture of the material grown and learned how to control key material properties such as microstructure, surface morphology (faceted versus evenly smooth), grain size (microcrystalline versus nanocrystalline), surface roughness (from rough $120 \mathrm{rms}$ to smooth $30 \mathrm{rms}$ ), and bonding configuration $\left(\mathrm{sp}^{3} \mathrm{C}\right.$ versus $\left.\mathrm{sp}^{2} \mathrm{C}\right)$.

\section{ACKNOWLEDGMENTS}

The corresponding author wishes to acknowledge the facilities housed in the Center of Applied Science and Engineering (CASE) directed by Dr. Ryan E. Giedd (MSU) and ONR Grant for financial support and Mr. J. Riley (undergraduate student) for technical assistance in Raman measurements.

\section{REFERENCES}

1. R. Kalish: Properties of Diamond, edited by G. Davies (INSPEC, 1994), pp. 79-91.

2. J.A. Garrido, C.E. Nebel, M. Stutzmann, E. Gheeraert, N. Casanova, E. Bustarret, and A. Deneuville: A new acceptor state in CVDdiamond. Diamond Relat. Mater. 11, 347 (2002).

3. J.C. Angus, P. Koidl, and S. Domitz: Plasma Deposited Thin Films, edited by J. Mort and F. Jansen (CRC, Boca Raton, FL, 1986).

4. P.K. Bachmann and R. Messier: Chem. Eng. News 67, 24 (1989).

5. M.H. Nazare: Properties and Growth of Diamond, edited by G. Davies (EMIS Data Review Series, INSPEC, 1994), p. 85.

6. P. John: The oxidation of (100) textured diamond. Diamond Relat. Mater. 11, 861 (2002).

7. J.B. Cui, J. Robertson, and W.I. Milne: The effect of film resistance on electron field emission from amorphous carbon films. Diamond Relat. Mater. 10, 868 (2001).

8. K.H. Chen, Y.L. Lai, L.C. Chen, J.Y. Wu, and F.J. Kao: Hightemperature Raman study in CVD diamond. Thin Solid Films 270, 143 (1995).

9. K.H. Chen, J.Y. Wu, L.C. Chen, C.C. Juan, and T. Hsu: Wide bandgap semiconductors and devices-state-of-the-art program on compound semiconductors. Electrochemical Soc. Proc. 95-21, 57 (1995).

10. W.A. Yarbrough and R. Messier: Chemical vapor deposited diamond films. Science 247, 688 (1990).

11. D.M. Gruen: Nanocrystalline diamond. Annu. Rev. Mater. Sci. 29, 211 (1999).

12. T. Sharda, M.M. Rahaman, Y. Nukaya, T. Soga, T. Jimbo, and M. Umeno: High compressive stress in nanocrystalline diamond films grown by microwave plasma chemical vapor deposition. Diamond Relat. Mater. 10, 352 (2001).

13. N.A. Morrison, S. Muhl, S.E. Rodil, A.C. Ferrari, M. Nesladek, W.I. Milne, and J. Robertson: The preparation, characterization and tribological properties of TA-C:H deposited using an electron cyclotron wave resonance plasma beam source. Phys. Status Solidi A 172, 79 (1999).

14. S. Jiao, A. Sumant, M.A. Kirk, D.M. Gruen, A.R. Krauss, and O. Auciello: Microstructure of ultrananocrystalline diamond films grown by microwave $\mathrm{Ar}-\mathrm{CH}_{4}$ plasma chemical vapor deposition with or without added $\mathrm{H}_{2}$. J. Appl. Phys. 90, 118 (2001).

15. G. Amaratunga: Watching the nanotube. IEEE Spectrum, Sept., 28 (2003).

16. A.V. Sumant, D.S. Grierson, J.E. Gerbi, J. Birrell, U.D. Lanke, O. Auciello, J.A. Carlisle, and R.W. Carpick: Toward the ultimate tribological interface: Surface chemistry and nanotribology of ultrananocrystalline diamond. Adv. Mater. 17, 1039 (2004).

17. A.R. Krauss, O. Auciello, D.M. Gruen, A. Jayatissa, A. Sumant, J. Tucek, D.C. Macini, N. Molodvan, A. Erdemir, D. Ersoy, M.N. Gardos, H.G. Busmann, E.M. Meyer, and M.Q. Ding: Ultrananocrystalline diamond thin films for MEMS and moving mechanical assembly devices. Diamond Relat. Mater. 10, 1952 (2001). 
18. J. Robertson: Diamond-like carbon. Philos. Mag. B 76, 335 (1997).

19. R. Kalish: The search for donors in diamond. Diamond Relat. Mater. 10, 1749 (2001).

20. S. Gupta, B.R. Weiner, and G. Morell: Investigations of the electron field emission properties and microstructure correlation in sulfur-incorporated nanocrystalline carbon thin films. J. Appl. Phys. 91, 10088 (2002).

21. S. Gupta, A. Martinez, B.R. Weiner, and G. Morell: Electrical conductivity studies of chemical vapor deposited sulfurincorporated nanocomposite carbon thin films. Appl. Phys. Lett. 81, 283 (2002).

22. S. Gupta, B.R. Weiner, and G. Morell: Role of $\mathrm{sp}^{2} \mathrm{C}$ cluster size on the field-emission properties of sulfur-incorporated nanocomposite carbon thin films. Appl. Phys. Lett. 80, 1471 (2002).

23. O.A. Williams, S. Curat, R.B. Jackman, J.E. Gerbi, and D.M. Gruen: $n$-type conductivity in ultrananocrystalline diamond films. Appl. Phys. Lett. 85, 1680 (2004).

24. S. Gupta, B.R. Weiner, and G. Morell: Electron field emission properties of microcrystalline and nanocrystalline carbon thin films deposited by S-assisted hot filament CVD. Diamond Relat. Mater. 11, 799 (2002).

25. G. Binnig, C.F. Quate, and C. Gerber: Atomic force microscope. Phys. Rev. Lett. 56, 930 (1986).

26. B.D. Cullity: Elements of X-Ray Diffraction, 2nd ed. (AddisonWesley, Boston, MA, 1978), pp. 102-111.

27. D.S. Knight and W.B. White: Characterization of diamond films by Raman spectroscopy. J. Mater. Res. 4, 385 (1989).

28. M. Yoshikawa: Properties and characterization of amorphous carbon films. Mater. Sci. Forum 52 \& 53, 365 (1989).

29. S. Gupta, R.S. Katiyar, D.R. Gilbert, R.K. Singh, and G. Morell: Microstructural studies of diamond thin films grown by electron cyclotron resonance-assisted chemical vapor deposition. J. Appl. Phys. 88, 5695 (2000).

30. L. Bergmann and R.J. Nemanich: Raman and photoluminescence analysis of stress state and impurity distribution in diamond thin films. J. Appl. Phys. 78, 6709 (1995).

31. R.J. Nemanich, J.T. Glass, G. Luckovsky, and R.E. Shröder: Raman scattering characterization of carbon bonding in diamond and diamond-like thin films. J. Vac. Sci. Technol., A 6, 1783 (1988).

32. S. Gupta, B.R. Weiner, and G. Morell: Synthesis and characterization of sulfur-incorporated microcrystalline diamond and nanocrystalline carbon thin films by hot filament chemical vapor deposition. J. Mater. Res. 18(2), 363 (2003).

33. O.A. Williams, M. Daenen, J.D. Haen, K. Haenen, M. Nesladek, and M.D. Olieslaeger, ADC05, Argonne National Laboratory, IL.

34. M. Dembo and Y.L. Wang: Stresses at the cell-to-substrate interface during locomotion of fibroblasts. Biophys. J. 76(4), 2307 (1999).

35. J. Domke, W.J. Parak, M. George, H.E. Gaub, and M. Radmacher: Mapping the mechanical pulse of single cardiomyocytes with the atomic force microscope. Eur. Biophys. J. 28, 179 (1999).

36. J. Domke, S. Dannohl, W.J. Parak, O. Muller, W.K. Aicher, and M. Radmacher: Substrate dependent differences in morphology and elasticity of living osteoblasts investigated by atomic force microscopy. Colloids Surf., B Biointerfaces 19, 367 (2000).

37. J.H. Hoh, W.F. Heinz, E. A-Hassan: Support Note No. 240 Part B Digital Instruments (1997).

38. C. Rotsch and M. Radmacher: Mapping local electrostatic forces with the atomic force microscope. Langmuir 13, 2825 (1997).

39. M. Chhowalla, A.C. Ferrari, J. Robertson, and G.A.J. Amaratunga: Evolution of $\mathrm{sp}^{2}$ bonding with deposition temperature in tetrahedral amorphous carbon studied by Raman spectroscopy. Appl. Phys. Lett. 76, 1419 (2000).

40. A.C. Ferrari and J. Robertson: Origin of the $1150-\mathrm{cm}^{-1}$ Raman mode in nanocrystalline diamond. Phys. Rev. B 63(12), 1405 (2001).

41. H. Kuzmany, R. Pfeiffer, N. Salk, and B. Günther: The mystery of the $1140 \mathrm{~cm}^{-1}$ Raman line in nanocrystalline diamond films. Carbon 42, 911 (2004). 